\title{
Review
}

\section{The Use of Herbal Medicine in Alzheimer's Disease-A Systematic Review}

\section{Leopoldo Luiz dos Santos-Neto ${ }^{1}$, Maria Alice de Vilhena Toledo ${ }^{2}$, Patrícia Medeiros-Souza ${ }^{3}$ and Gustavo Almeida de Souza ${ }^{3}$}

\author{
${ }^{1}$ General Internal Medical Center, University Hospital of Brasilia, University of Brasilia, ${ }^{2}$ General Internal Medical \\ Center, Department of Geriatrics, University Hospital of Brasilia, University of Brasilia and ${ }^{3}$ School of Pharmacy, \\ Department of Health Sciences, University of Brasilia, Brazil
}

\begin{abstract}
The treatments of choice in Alzheimer's disease (AD) are cholinesterase inhibitors and NMDA-receptor antagonists, although doubts remain about the therapeutic effectiveness of these drugs. Herbal medicine products have been used in the treatment of Behavioral and Psychological Symptoms of Dementia (BPSD) but with various responses. The objective of this article was to review evidences from controlled studies in order to determine whether herbs can be useful in the treatment of cognitive disorders in the elderly. Randomized controlled studies assessing AD in individuals older than 65 years were identified through searches of MEDLINE, LILACS, Cochrane Library, dissertation Abstract (USA), ADEAR (Alzheimer's Disease Clinical Trials Database), National Research Register, Current Controlled trials, Centerwatch Trials Database and PsychINFO Journal Articles. The search combined the terms Alzheimer disease, dementia, cognition disorders, Herbal, Phytotherapy. The crossover results were evaluated by the Jadad's measurement scale. The systematic review identified two herbs and herbal formulations with therapeutic effects for the treatment of AD: Melissa officinalis, Salvia officinalis and Yi-Gan San and BDW (Ba Wei Di Huang Wan). Ginkgo biloba was identified in a meta-analysis study. All five herbs are useful for cognitive impairment of AD. M. officinalis and Yi-Gan San are also useful in agitation, for they have sedative effects. These herbs and formulations have demonstrated good therapeutic effectiveness but these results need to be compared with those of traditional drugs. Further large multicenter studies should be conducted in order to test the cost-effectiveness of these herbs for $\mathrm{AD}$ and the impact in the control of cognitive deterioration.
\end{abstract}

Keywords: Alzheimer's disease - cognitive impairment - dementia - elderly - herbs - randomized clinical trial - systematic review

\section{Introduction}

Alzheimer's disease (AD) is characterized as a progressive neurodegenerative disorder and considered as prominent cause of dementia in the elderly. The main characteristics of this disease are difficulties in household handling routine and cognitive and emotional disturbance in the elderly. The

For reprints and all correspondence: Leopoldo Luiz dos Santos-Neto, Centro de Clínica Médica, Hospital Universitário de Brasília (HUB)-UnB, Caixa postal 04438, 70919-970 Brasília-DF, Brazil. Tel: +61-81610333;

Fax: +61-32451875; E-mail: leoneto@ uninet.com.br treatment of $\mathrm{AD}$ is a clinical challenge. With the development of cholinesterase inhibitors and a $N$-methyl-D-aspartate antagonist (memantine), good perspectives have emerged in controlling the symptoms of AD. Therapeutic decisions have to be guided by clinical studies and should consider the physiopathogenesis and epidemiology of the disease.

The main objective of these clinical trials is to reduce the Behavioral and Psychological Symptoms of Dementia (BPSD) and to improve cognition and the functional activity status, thus reducing the impairment of instrumental activities of the daily living (IADLs) and to lower the institutionalization rates (nursing home placement). Unfortunately, only a limited 
number of trials have dealt with this topic and with follow-up periods shorter than two years. In spite of the absence of sufficient therapeutic effectiveness in mild and moderate $A D$, these drugs are still considered as the first line of treatment for AD (1). Studies of cost-effectiveness suggest that memantine $(2,3)$ and donepezil (4) are useful in the reduction of institutionalized care and/or cognitive impairment in patients with AD. Recently, two clinical trials showed no improvement of the cognitive deficit $(5,6)$ or reduction in the institutionalization rate (6).

Searching for alternatives, many herbal products have been tested and employed in the treatment of $\mathrm{AD}$, but with different clinical responses (7). The assessment of these drugs through randomized controlled trials should be useful to identify effective products in the treatment of $\mathrm{AD}$.

\section{Methods}

Searching at MEDLINE (during April 2006, PubMed), LILACS (Latin American and Caribbean Health Science Literature: 40th edition, May 2001, the last research was performed in April 2006); Cochrane Library (issue 1, 2006); Dissertation Abstract (USA, during April 2006); ADEAR (Alzheimer's Disease Clinical Trials Database, until April 2006); National Research Register (1/2006); Current Controlled trials (the last research was performed in October 2005); PsychINFO Journal Articles (during the year of 2006); relevant web sites; and scanning of reference list of relevant articles. There were no language or publication restrictions.

Search for keywords in MeSH (medical subject heading (MeSH) with the words "Alzheimer disease, dementia, cognition disorders' was performed first. In the second part, the keywords were 'Herbal' and 'Phytotherapy'. The crossover results of the two searches were evaluated by the Jadad's measurement scale (8).

Inclusion criteria: Three investigators independently reviewed all of the articles found. The articles were selected using the criteria listed below:

i. The studies should be randomized; double-blind and controlled (with a control group and a treatment group).

ii. Studies should establish methodological procedures in the crossover or be conducted at the same time.

iii. In the case of being crossover, a washout period of at least 7 days was required.

iv. Patients included in the researches had their diagnosis rated into three degrees as follows: mild, moderate and severe forms of $\mathrm{AD}$, according to the criteria from the National Institute of Neurological and Communicative Disorders and Stroke-AD and Related Disorders Association (NINCDS-ADRDA) (9). The models used were as follows: Mini-mental beginning values between 10 and 26 (initial and mild group) or $<10$ (initial group).

v. Clinical trials should last for at least 1 month (4 weeks). vi. Detailed description of the herbal product used.

vii. Neuropsychiatry symptoms progression should be measured with numerical score using the Assessment Scale (ADAS-noncog, range of score, 0-70), NPI (Neuropsychiatric Inventory, range of score 0-120), Clinical Global impression of change or Behavioral rating scale for Geriatric patients.

viii. The final score should be quantified using a combination of ADL and IADL methodological procedures.

Exclusion criteria: The herbal product has already been target of a quantified systematic review study. In this case, only the results of the studies will be considered.

Jadad's measurement scale: Methodological quality was assessed using a scale developed and validated by Jadad et al. (8). This scale assesses the completeness of reporting using three items with a five points maximum score. If the allocation into groups is explicitly randomized, item 1 is scored. A bonus point is given if an adequate method to generate the random sequence is described. If there is an explicit statement that the study is double-blind Item 2 is scored. A bonus point is given if the method is described and adequate. Item 3 is scored if there is either an explicit statement that all patients included were also analyzed or if the number and reasons for dropouts in all groups are given separately. For being classified as adequately reported a trial should score at least three of five points, a cut-off point is recommended by the author of the scale (10).

All extraction and quality assessments were performed by at least two independent reviewers using standard forms developed for each review. Disagreements were documented and discussed with final decisions made by the principal reviewer.

\section{Results}

Two herbs and two herbal formulations were identified to have effectiveness in the treatment of cognitive disturbance of AD in the systematic review: Salvia officinalis (11), Melissa officinalis (12), and Yi-Gan San (13) and Ba Wei Di Huang Wan (BDW) (14). The main characteristics of the study are described in Table 1.

Gingko biloba was previously identified in one metaanalysis (15), and only the conclusions of the study will be considered. Another study will be conducted with huperzine A, a product derived from a Chinese herb Huperzia serrata, to evaluate the safety and efficacy in the treatment of AD in a multicenter randomized controlled trial of its effect on cognitive function (16).

The studies of Salvia (11), Melissa (12), Yi-Gan San (13) and BDW (14) have reached Jadad's measurement scale of $\geq 3$. The researches had a follow up of 1 month (Yi-Gan San) (13), 2 months (BDW) (14) and 4 months (Salvia and Melissa) $(11,12)$. All samples studied were composed of patients with initial mild symptoms judged as AD. Two studies compared herbal medicines and control samples, using intention to treat [Salvia (11) and Melissa (12)]. 


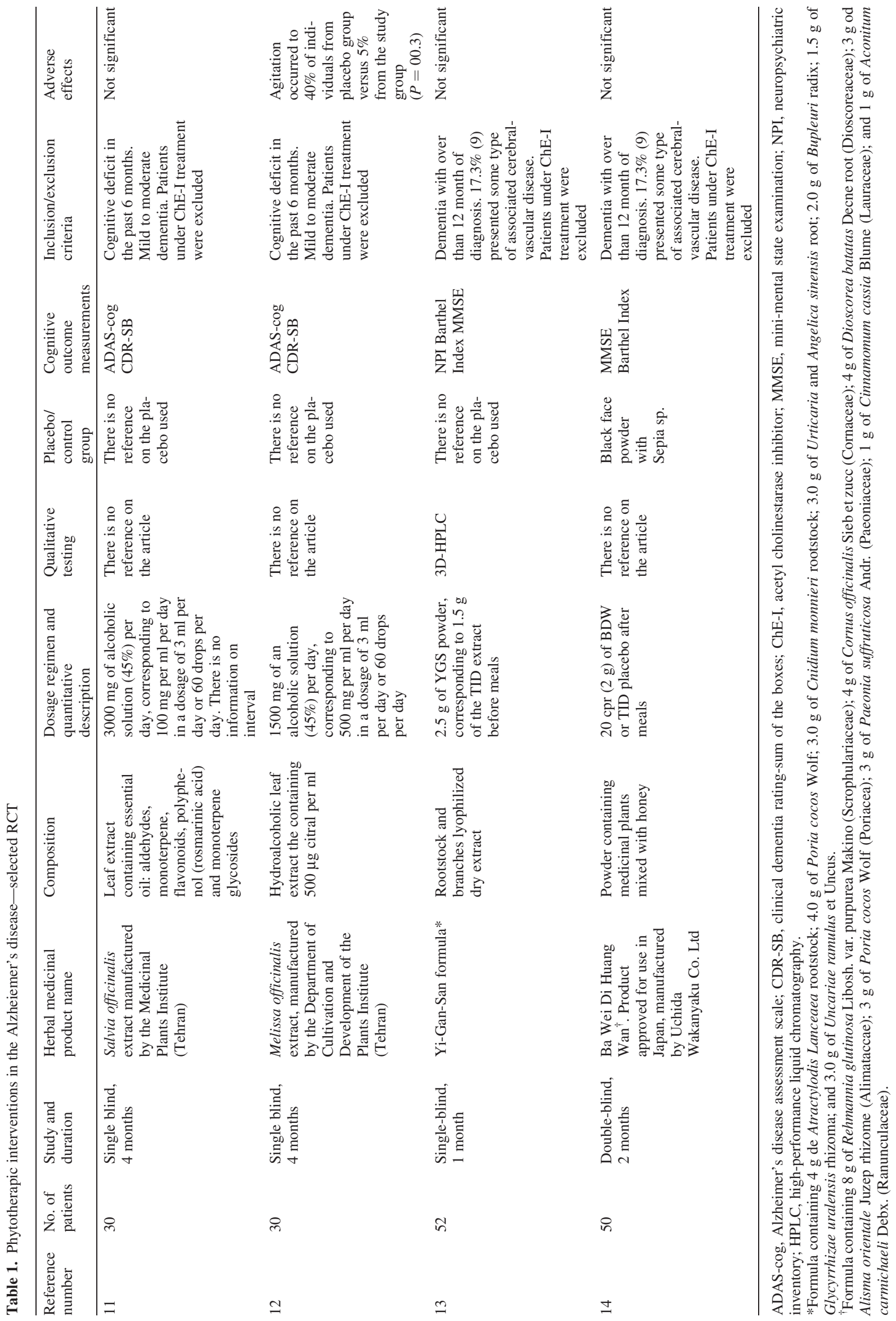


None of the studies evaluated the institutionalization rate or compared the active principle with the current therapies with Acetyl Cholinesterase Inhibitor or memantine.

\section{Discussion}

The results of this systematic review identified four studies with methodological quality assessing $S$. officinalis (11), M. officinalis (12), Yi-Gan San (13) and BDW (14). The last two are composed of formulations with different phytoactive agents. These herbs and formulations presented efficiency in reducing the mild and moderate symptoms of $\mathrm{AD}$.

Gingko biloba presented statistically significant mild effectiveness in the treatment of cognitive deficit in AD. The meta-analysis study of Cochrane (13) concluded that additional controlled studies would be necessary in order to recognize cognitive improvement with the use of gingko. There is still need of a prospective study with an appropriate duration and representative sample to identify if $G$. biloba reduces the development of $\mathrm{AD}$ (17). Another plant with a large application perspective is $H$. serrata, after multicenter trial confirmation underway (16).

Melissa and Yi-Gan San showed reduction in the cognitive deficits and a good sedative effect in patients with $\operatorname{AD}(12,13)$. Previous clinical studies showed that the extract of M. officinalis reduces laboratory-induced stress (18) and might have benefits in mood improvement (19-21). The use of these herbs and formulations should be well tolerated, (22) and adverse effects have not yet been reported (23). Further studies should be conducted to compare the current therapies for $\mathrm{AD}$ and the use of these herbal remedies in controlling the symptoms of AD.

The action mechanisms of these herbs and formulations are not well known. It has been suggested that the chemical composition of the essential oil of the Melissa and Salvia leaf extracts are monoterpene aldehydes, polyphenol flavonoids (including rosmarinic acid) (24) and monoterpene glycosides (25). All of these components have many observable effects in vitro, which include powerful anti-oxidative activity $(26,27)$ and an affinity to nicotinic and muscarinic receptor in the human cerebral cortex (28). This last mechanism is of special interest, as modulation of cholinergic systems should play a role in improving the cognitive function, especially in AD.

Yi-Gan San and BDW are mixes of many herbal ingredients. Yi-Gan San is a mixture of 7 different dried plants, many of them (Unticariae sinensis and Angelicae root) with possible actions in the serotoninergic and gaba system. BDW consists of 8 herbs: Rehmannia glutinosa Libosh. var purpurea Makino, Cornus officinalis Sieb et Zucc (Cornaceae), Dioscorea batatas Decne root (Dioscoreaceae), Alisma orientale Juzep rhizome (Alimataccae), Poria cocos Wolf, Paeonia suffruticosa Andr. (Paeoniaceae), Cinnamomum cassia Blume (Lauraceae) and Aconitum carmichaeli Debx. (Ranunculaceae). Studies have suggested that BDW enhances the choline acetyltransferase activity and increases the acetylcholine content of the frontal cortex in a murine model $(29,30)$.
Major methodological limitations of the four studies are small size of samples and short-term duration. There is no description of the chemical composition and/or possible active principles of the different products employed in studies Involving Yi-Gan San (13) and BDW (14) formulation (Table 1). In the study with BDW (13), Sepia sp. and face powder were used as placebo; however, the way that the shellfish formulation was performed was not described. In the control group of the study using the Yi-Gan-San (14) formulation, $25 \mathrm{mg}$ per day of tiapride hydrochloride were introduced. This drug is a substituted derivative with selective dopamine D2-receptor antagonist properties. This intervention (contamination) occurred in $44 \%$ of the individuals and was responsible for the symptoms, among them dizziness.

Generally, crude herbal drugs are natural products and their chemical composition depends on several factors such as geographic source of the plant material, climate in which it was grown, and time of harvest. Commercially available herbal medicinal products also vary in their content and concentration of chemical constituents from batch to batch and when products containing the same herbal ingredient are compared between manufacturers. Even when herbal products are standardized for content of known active or marker compounds to achieve more consistent pharmaceutical quality, variations in the concentrations of other constituents can be observed. The use of a protocol such as the Consolidated Standards of Reporting Trials (CONSORT), composed of 22 items, will probably minimize the limitations of RTC with phytotherapic agents (31).

The use of herbal medicines in the treatment of AD should be compared with the pharmacological treatment currently in use. Such studies should include the identification of the active principle in order to improve the validation of the clinical trial. Further large-scale, multicenter studies are necessary to determine the effectiveness of these substances in the cognitive deterioration of AD. Until then, this review provides some evidence of the benefit of Melissa, Salvia, Yi-Gan San and $\mathrm{BDW}$ in the treatment of AD.

\section{References}

1. Doody RS, Stevens JC, Beck C, Dubinsky RM, Kaye JA, Gwyther L, et al. Practice parameter: management of dementia (an evidence-based review). Neurology 2001;56:1154-66.

2. Jones RW, McCrone P, Guilhaume C. Cost effectiveness of memantine in Alzheimer's disease. An analysis based on a probabilistic Markov model from a UK perspective. Drugs Aging 2004;21:607-20.

3. François C, Sintonen H, Sulkava R, Rive B. Cost effectiveness of Memantine in moderately severe Alzheimer's' Disease. A Markov model in Finland. Clin Drug Invest 2004;24:373-84.

4. Feldman H, Gauthier S, Hecker J, Vellas B, Hux M, Xu Y, et al. Economic evaluation of donezepil in moderate to severe Alzheimer disease. Neurology 2004;63:644-50.

5. Salloway S, Ferris S, Kluger A, Goldman R, Griesing T, Kumar D, et al. Efficacy of donepezil in mild cognitive impairment. A randomized placebo-controlled trial. Neurology 2004;63:651-7.

6. AD2000 Collaborative Group. Long-term donepezil treatment in 565 patients with Alzheimer's disease (AD2000): randomized double-blind trial. Lancet 2004;363:2105-15.

7. Mantle D, Pickering AT, Perry E. Medical Plant extracts for treatment of dementia. A review of their pharmacology, efficacy and tolerability. CNS Drugs 2000;13:201-13. 
8. Jadad AR, Moore RA, Carroll D, Jenkinson C, Reynolds DJ, Gavaghan DJ, et al. Assessing quality of reports of randomized clinical trials: is blinding necessary? Control Clin Trials 1996;17:1-12.

9. McKhann G, Drachman D, Folstein M, Katzman R, Price D, Stadlan EM. Clinical diagnosis of Alzheimer's disease: report of the NINCDS-ADRDA Work Group under the auspices of Department of Health and Human Services Task Force on Alzheimer's Disease. Neurology 1984;34:939-44.

10. Khan KS, Daya S, Jadad AR. The importance of quality of primary studies in producing unbiased systematic reviews. Arch Intern Med 1996;156: 661-6.

11. Akhondzadeh S, Noroozian M, Mohammadi M, Ohadinia S, Jamshidi AH. Salvia officinalis extract in the treatment of patients with mild to moderate Alzheimer's disease: a double blind, randomized and placebo-controlled trial. J Clin Pharm Ther 2003;28:53-9.

12. Akhondzadeh S, Noroozian M, Mohammadi M, Ohadinia S, Jamshidi AH, Khani M. Melissa officinalis extract in the treatment of patients with mild to moderate Alzheimer's disease: a double blind, randomized and placebocontrolled trial. J Neurol Neurosurg Psychiatr 2003;74:863-6.

13. Iwasaki K, Satoh-Nakagawa T, Maruyama M, Monma Y, Nemoto M, Tomita $\mathrm{N}$ e cols. A randomized, observer-blind, controlled trial of the traditional Chinese medicine Yi-Gan San for improvement of behavioral and psychological symptoms and activities of daily living in dementia patients. J Clin Psychiatr 2005;66:248-52.

14. Iwasaki K, Kobayashi S, Chimura Y, Taguchi M, Inoue K, Cho S e cols. A randomized, double-blind, placebo-controlled clinical trial of the Chinese herbal medicine 'Ba Wei Huang Wan' in the treatment of dementia. $J$ Am Geriatr Soc 1994;52:1518-21.

15. Birks J, Grimley EJ. Ginkgo biloba for cognitive impairment and dementia. The Cochrane Library, Issue 2, 2004. Oxford: Update Software.

16. http://clinicaltrials.gov/ct/how/NCT00083590?order=9.

17. DIGGER. Dementia in General practice-Ginkgo Extract Research trial., http://www.controlled-trials.com/

18. Kennedy DO, Little W, Scholey AB. Attenuation of laboratory-induced stress in humans after acute administration of Melissa officinalis (lemon balm). Psychosom Med 2004;66:607-13.

19. Kennedy DO, Scholey AB, Tildesley NTJ, Perry EK, Wesnes KA. Modulation of mood and cognitive performance following acute administration of Melissa officinalis (lemon balm). Pharmacol Biochem Behav 2002;72:953-64.

20. Kennedy DO, Wake G, Savelev S, Tildesley NT, Perry EK, Wesnes KA, et al. Modulation of mood and cognitive performance following administration of single doses of Melissa officinalis (Lemon balm) with human CNS nicotinic and muscarinic receptor binding properties. Neuropsychopharmacology 2003;28:1871-81.

21. Ballard C, O'Brien J, Reichelt K, Perry E. Aromatherapy as a safe and effective treatment for the management of agitation in severe dementia: the results of a double blind, placebo controlled trial. J Clin Psychiatr 2002;63:553-8.

22. Cerny A, Schmid K. Tolerability and efficacy of valerian/lemon balm in healthy volunteers: a double-blind, placebo-controlled, multicenter study. Phytoterapia 1999;70:221-8.

23. Wong AHC, Smith M, Boon HS. Herbal remedies in psychiatric practice. Arch Gen Psychiatr 1998;55:1033-44.

24. Carnat AP, Carnat A, Fraisse D, Lamaison JL. The aromatic and polyphenolic composition of lemon balm (Melissa officinalis L. subsp. officinalis) tea. Pharm Acta Helv 1998;72:301-5.

25. Mulkens A, Stephanou E, Kapetenadis I. Heterosides a genines volatiles dans les feuilles de Melissa officinalis L. (lamiaceae). Pharm Acta Helv 1985;60:276-8.

26. Mantle D, Eddeb F, Pickering AT. Comparison of relative antioxidant activities of British medicinal plant species in vitro. J Ethnopharmacol 2000;72:47-51.

27. Hohmann J, Zupko I, Redei D, Csanyi M, Falkay G, Mathe I, et al. Protective effects of the aerial parts of Salvia officinalis, Melissa officinalis and Lavandula angustifolia and their constituents against enzyme-dependent and enzyme independent lipid peroxidation. Planta Med 1999;65:576-8.

28. Wake G, Court J, Pickering A, Lewis R, Wilkins R, Perry E. CNS acetylcholine receptor activity in European medicinal plants traditionally used to improve failing memory. $J$ Ethnopharmacol 2000;69:105-114.

29. Hirokawa S, Nose M, Amagaya S, Oyama T, Ogihara Y. Protective effect of hachimi-jio-gan, an oriental herbal medicinal mixture, against cerebral anoxia. J Ethnopharmacol 1993;40:201-6.

30. Hirokawa S, Nose M, Ishige A, Amagaya S, Oyama T, Ogihara Y. Effect of Hachimi-jio-gan on scopolamine-induced memory impairment and on acetylcholine content in rat brain. J Ethnopharmacol 1996;50:77-84.

31. Gagnier JJ, Boon H, Rochon P, Moher D, Barnes J, Bombardier C. CONSORT Group. Reporting randomized, controlled trials of herbal interventions: an elaborated CONSORT statement. Ann Intern Med 2006;144:364-7

Received November 26, 2005; accepted September 15, 2006 


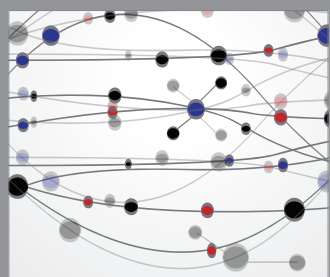

The Scientific World Journal
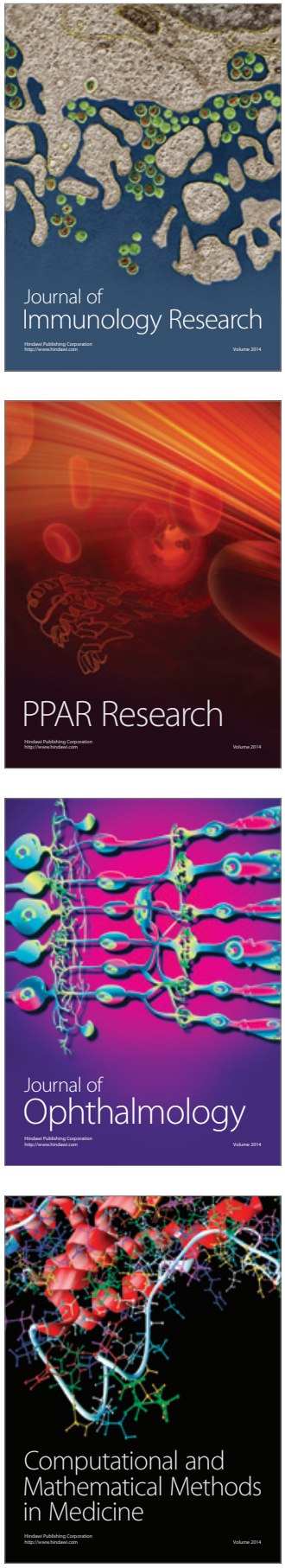

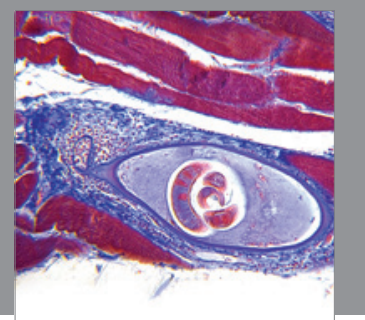

Gastroenterology

Research and Practice
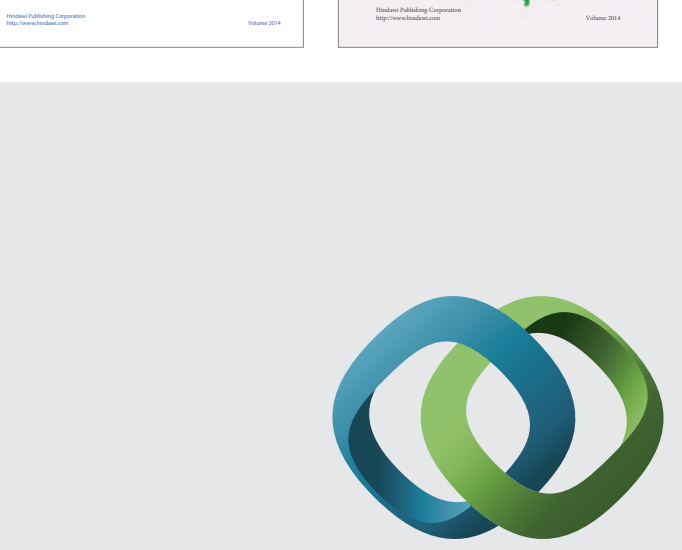

\section{Hindawi}

Submit your manuscripts at

http://www.hindawi.com
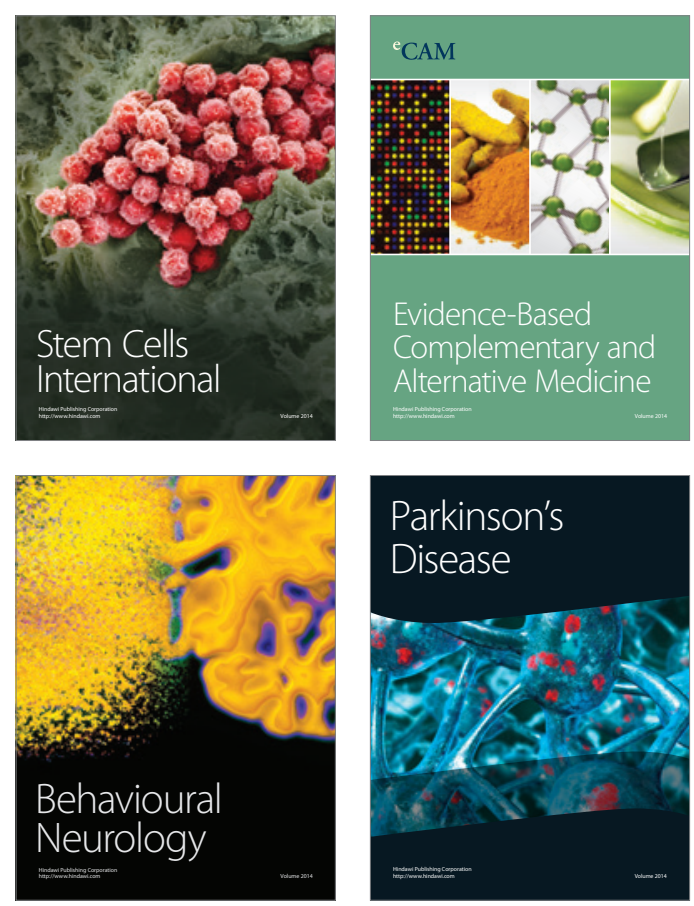

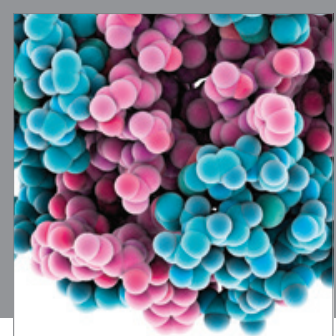

Journal of
Diabetes Research

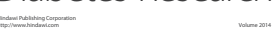

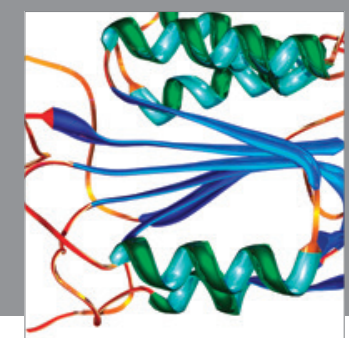

Disease Markers
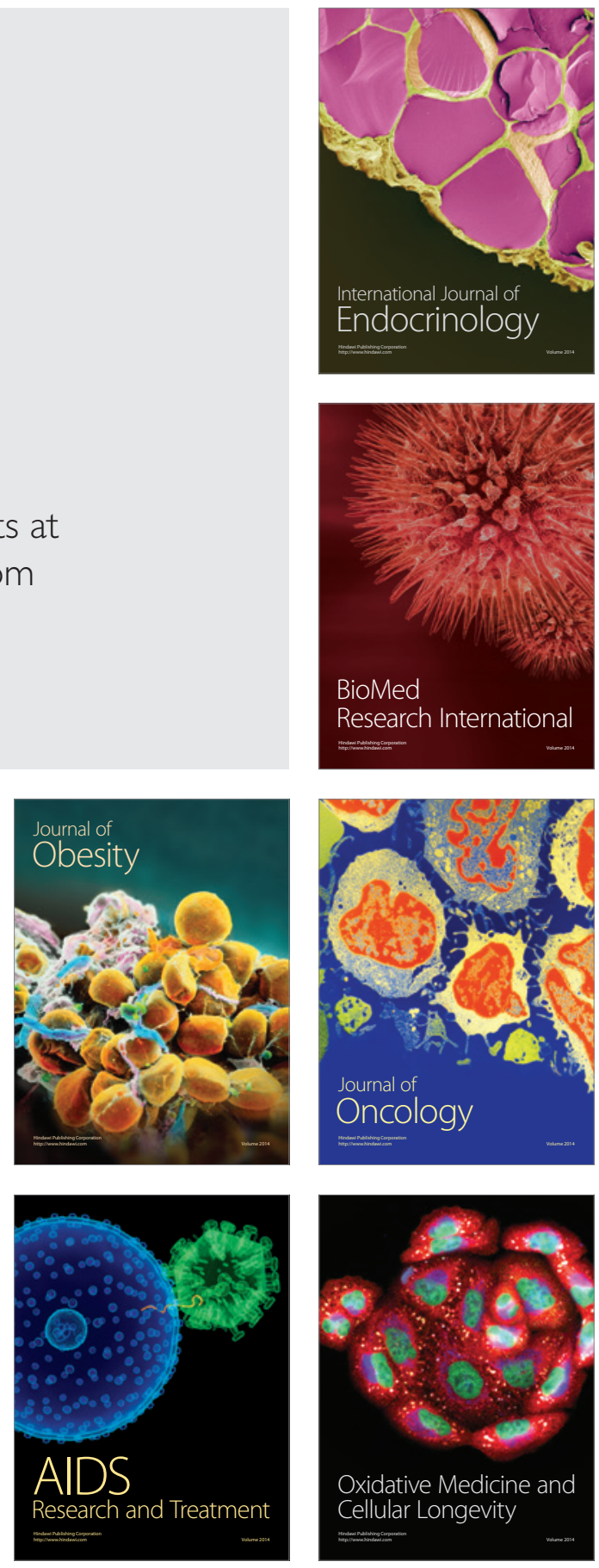